\title{
VALORES DE REFERÊNCIA PARA MACRONUTRIENTES EM EUCALIPTO OBTIDOS PELOS MÉTODOS DRIS E CHANCE MATEMÁTICA ${ }^{(1)}$
}

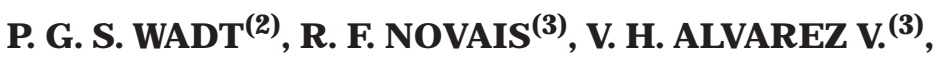 \\ S. FONSECA ${ }^{(4)} \&$ N. F. BARROS(3)
}

\begin{abstract}
RESUMO
Informações de 1.213 árvores de plantios comerciais foram obtidas, entre os anos de 1988 a 1994, de híbridos de Eucalyptus grandis x E. urophylla, da Aracruz Celulose S.A., cultivados no estado do Espírito Santo, Brasil. Os resultados da produti vidade e concentração dos macronutrientes em quatro órgãos das árvores foram utilizados na obtenção dos valores de referência para a concentração dos nutrientes, pelos métodos da Chance Matemática e do DRIS. E mpregou-se, no cálculo dos índices DR IS, a fórmula de J ones (1981), a divisão da população-base em estratos de idade e a seleção das relações entre os nutrientes pelo teste F. 0 nível crítico dos nutrientes foi atingido pelo DRIS por meio de dois critérios: pela concentração média dos nutrientes nos talhões nutricionalmente balanceados e produtivos (NCO) e pelo critério gráfico (NCG). 0 primeiro critério mostrou-se mais promissor, inclusive em relação ao método da Chance Matemática, o qual foi adequado principalmente para a determinação de faixa óti ma que poderia ser adotada como referência para o limite inferior e o superior de árvores com níveis adequados dos nutrientes.
\end{abstract}

Termos de indexação: eucalipto, nível crítico, faixa de suficiência, índices DRIS.

\section{SUMMARY: REFERENCE VALUES OF MACRONUTRIENTS IN EUCALYPT DETERMINED BY DRIS AND MATHEMATICAL CHANCE METHODS}

Data from 1.213 trees of Eucalyptus grandis x E. urophylla hybrids were collected between 1988 and 1994 from theAracruz Celul oseS.A. commercial plantation in theState

(1) Recebido para publicação em março e aprovado em agosto de 1998.

(2) D.S. META AGROF LORESTAL. Chácara Primavera. Caixa Postal 224, CEP 13730-000 Mococa (SP). E-mail: pgswadt@dgl net.com.br.

(3) Professor do Departamento de Solos, Universidade Federal de Viçosa, CEP 36571-000 Viçosa (MG). Bolsita do CNPq. E-mail: nfbarros@mail.ufv.br.

(4) M.S. Pesquisador Sênior da ARACRUZ CELULOSE S.A., Rod. Aracruz Barra do Riacho, Km 25, CEP 29190-000 Aracruz (ES). 
of Espírito Santo, Brazil. Data on stand productivity and macronutrient concentration in four tree components (leaf, branch, stembark and stemwood) were used to determine the reference values of nutrient concentration by the methods of Mathematical Chance and DRIS. The formula of $J$ ones and the population distribution based on age and sel ection of the relation between elemental concentrations by the $F$ test were used in the calculation of DRIS indices. Critical level s wereobtained using theDRIS method, by thefollowing processes: determining the mean nutrient concentration in the nutritionally balanced and productive (optimum critical level) eucalypt stands; and by graphs (critical-level graph). The mean concentration proved to bea promising method, even when compared to the Mathematical Chance method. This latter method was appropriate mainly for the determination of the optimum range, which could be used as minimum and maximum limits for the range of nutrient sufficiency in productivetrees.

Index terms: Eucalyptus, optimum levels, critical levels, sufficiency range

\section{INTRODUÇÃO}

A identificação do estado nutricional depende do estabel ecimento de valores de referência para a concentração dos nutrientes, tais como o nível crítico, o nível ótimo e a faixa ótima para a concentração dos nutrientes nas fol has ou noutros órgãos das árvores.

Convencionalmente, esses valores de referência são estabelecidos em experimentos de calibração, onde as características genéticas, ambientais e as interações entre os nutrientes são controladas (Bhargava \& Chadha, 1988). Por essa razão, os valores assim obtidos devem ser aplicados na avaliação de plantas crescidas sob as mesmas condições utilizadas no ensaio, o que torna o processo extremamente restritivo para uso em larga escala.

Além disto, esses valores de referência não são definitivos, estando sujeitos a revisões periódicas em conseqüência da introdução de novos materiais, novas técnicas silviculturais ou do cultivo em novos ambientes, o que exigiria, periodicamente, a instalação de experimentos de calibração, os quais, para o eucalipto, serão sempre de longa duração.

U ma alternativa aos experimentos de cal ibração refere-se ao aproveitamento de informações de monitoramento nutricional desenvolvido em tal hões comerciais. Estas informações agregam dados oriundos de ampla variação ambiental e, portanto, não podem ser utilizadas para determinar curvas de resposta como aquel as obti das nos experimentos de cali bração. Contudo, é possível utilizar os métodos do DRIS e da Chance Matemática para a obtenção dos valores dereferência a partir dessas informações. Por exemplo, Oliveira \& Sousa $(1988,1993)$ utilizaram o DRIS com o propósito de determinar os níveis de suficiência para os teores dos macronutrientes e micronutrientes no solo, em arroz e soja, respectivamente.
Needham et al. (1990) propuseram, para Pinus taeda L., o uso do DRIS para a determinação do nível crítico dos nutrientes. A vantagem do DRIS reside na identificação das árvores nutricionalmente equilibradas para todos os nutrientes, de forma a obter os valores ótimos para a concentração dos nutrientes, além de o DRIS levar em consideração as interações entre el es.

Mais recentemente, Wadt et al. (1994) sugeriram o método da Chance Matemática para determinar os limites de suficiência para a concentração dos nutrientes, oqual foi aplicadoem Coffea canephora L . (Novais et al., 1994).

O objetivo deste trabalho foi determinar os valores de referência para a concentração dos nutrientes nas árvores por critérios baseados no DRIS e no método da Chance Matemática, a partir de informações oriundas de monitoramento nutricional de plantações comerciais de eucalipto.

\section{MATERIAL E MÉTODOS}

\section{População-base}

Foram utilizadas informações de 1.213 árvores adultas (idade acima de cinco anos), de talhões comerciais de híbridos de Eucalyptus grandis $x \mathrm{E}$. urophylla, do estado do Espírito Santo (Brasil). As informações, coletadas entre os anos de 1988 e 1994, consistiram da produtividade (incremento médio

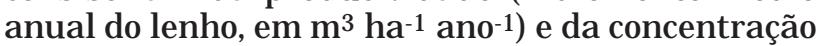

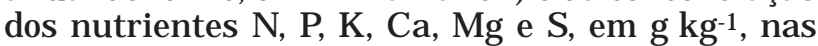
folhas, galhos, casca e lenho. A esse conjunto de informações denominou-se população-base.

\section{Obtenção das normas DRIS}

A população-base foi estratificada por idade, considerando meio ano deampl itudee, depois, dentro 
de cada estrato, determinou-se a classificação das árvores segundo a produtividade. Conforme a produtividade das árvores, obtiveram-se três subpopulações, com vistas a representar um conjunto de árvores com baixa, média e alta produtividade, e denominadas, respectivamente, subpopulação de baixa, média e alta produtividade.

Definiu-sea subpopulação demédia produtividade a constituída de árvores, cuja produtividadefoi igual à média $\pm 0,5$ desvios-padrão das produtividades da população-base, em cada estrato de idade. As demais subpopulações foram constituídas de árvores, cuja produtividade ficou acima (alta produtividade) ou abaixo (baixa produtividade) desses limites.

As normas DRIS (estatísticas da população de referência) cal culadas foram a média, desvio-padrão e número de casos para as relações entre dois nutrientes, em cada um dos órgãos das árvores (fol has, gal hos, casca elenho) e para cada uma das subpopulações.

\section{Cálculo dos índices DRIS}

Para o cál culo dos índices, utilizou-se a fórmula de J ones (1981):

$$
f(X / Y)=[(R-r) / s]
$$

em que:

$\mathrm{R}=$ val or da relação $\mathrm{X} / \mathrm{Y}$ entre as concentrações dos nutrientes $X$ e $Y$, em determinado órgão da árvore;

$r=$ valor médio das relações $X / Y$ da população de referência (norma média);

$\mathrm{s}=$ desvio-padrão das relações $X / Y$ da população de referência (norma desvio-padrão) e

$f(X / Y)=$ função da relação $X / Y$.

$\mathrm{X}$ eY =concentração dos macronutrientes, sendo " $X$ " e "Y" a concentração de dois quaisquer nutrientes, desde que os nutrientes " $X$ " e " $Y$ " sejam distintos entre si.

Os índices DRIS, de cada nutriente, foram determinados pelo cálculo da média aritmética das funções $f(X / Y)$. O índice de balanço nutricional (IBN) foi obtido pelo somatório, em módulo, dos índices DRIS de cada órgão, tomados independentemente.

As relações $X / Y$, cuja variância diferiu a $10 \%$ de significância entrea subpopulação de al ta e de baixa produtividade, foram aproveitadas no cálculo dos índices DRIS, e as demais foram descartadas.

\section{Análise estatística dos índices DRIS}

Foram testadas duas hipóteses para validar os resultados dos índices DRIS. Ambas as hipóteses foram baseadas na freqüência, em contagem, do número de vezes em que cada nutriente ocorreu como o mais limitantepor defi ciência. Um nutriente qual quer foi considerado como o mais limitante por deficiência, quando, num órgão da árvore, tendo um índice DRIS negativo, este foi o de menor valor em relação aos índices DRIS dos demais nutrientes no mesmo órgão da árvore. Utilizou-se o teste de quiquadrado para aval iar se as freqüências observadas foram iguais às esperadas, em cada uma das hipóteses descritas a seguir.

N1 (hipótese nula 1): Esta hipótese considerou que a freqüência com que cada nutriente ocorreu como o mais limitante foi atribuída ao acaso. Sendo verdadeira a hipótese nula, as freqüências observadas para todos os nutrientes seriam estatisticamente iguais entre si. A freqüência esperada foi igual ao número total de observações dentro de cada dasse de produtividade, dividido pelo total de nutrientes analisados. A freqüência observada (FO) foi a contagem do número de vezes em que cada nutriente ocorreu como o mais limitante, em cada classe de produtividade e órgão da árvore.

N2 (hipótese nula 2): Esta hipóte considerou que a freqüência com que cada nutriente ocorreu como o mais limitante foi independente da dasse de produtividade das árvores. Sendo verdadeira a hipótese nula, a freqüência observada para os nutrientes na classe de média produtividade seria igual à freqüência observada nas demais dasses de produtividade. Assim, a freqüência esperada foi igual à freqüência de ocorrência dos nutrientes como os mais limitantes por deficiência na classe de média produtividade. A freqüência observada foi determinada para a ocorrência dos nutrientes como os mais limitantes por deficiência nas classes de baixa e de alta produtividade.

\section{Determi nação dos valores de referência pelo DRIS}

Foram determinados os valores de referência para a concentração dos nutrientes N, P, K, Ca, Mge $\mathrm{S}$, nas fol has, gal hos, casca e lenho, pelo método do DRIS, a partir de dois procedimentos distintos, denominados, nível crítico ótimo (NCO) enível crítico gráfico (NCG). Ambos os processos foram descritos por Needlam et al. (1990). Na determinação do NCO e do NCG, utilizou-se o DRIS, para identificar, no conjunto de árvores de alta produtividade, aquelas que apresentaram mel hor condição nutricional .

Para cada nutriente em separado, foram considerados como em adequado estado nutricional aquelas árvores cujo IBN foi menor que a média $+1,33$ desvios-padrão dos IBN de todas as árvores do banco de dados e cujo índice DRIS do nutriente, em determinado órgão, estivesse no intervalo dado pelo valor médio $\pm 1,33$ desvios-padrão dos índices DRIS para o respectivo nutriente, no conjunto de árvores da população-base.

Finalmente, o NCO foi determinado pela média aritmética da concentração do nutriente neste conjunto de árvores que apresentaram melhor condição nutricional para cada nutriente. 
O NCG foi determinado pela interseção da curva deresposta no eixoY, da equação quadrática ajustada entre as concentrações do nutriente (tomadas como variável dependente) e os respectivos índices DRIS do nutriente (tomados como variável independente), para cada órgão das árvores que apresentaram melhor condição nutricional. A obtenção do NCG corresponde, grosso modo, à la aproximação no método descrito por Oliveira \& Sousa (1993) para determinar os níveis de suficiência, tendo esses autores desenvolvido outros procedimentos para indicar o nível de suficiência em função da produtividade desejada, por eles chamada de $2 a$ aproximação.

\section{Determinação dos valores de referência pela Chance Matemática}

Determinaram-se os val ores da faixa ótima e da mediana da faixa ótima para a concentração dos nutrientes $\mathrm{N}, \mathrm{P}, \mathrm{K}, \mathrm{Ca}, \mathrm{Mg}$ e $\mathrm{S}$ nas fol has, galhos, casca e lenho das árvores de eucalipto, pelo método da Chance Matemática (Wadt et al., 1994; Wadt, 1996).

Para tanto, inicialmente, determinou-se a amplitude (A) da concentração de dado nutrienteem determinado órgão; a seguir, calculou-se o número de classes possíveis (I) com base no tamanho da amostra ( $n$ ), em que I $=n^{1 / 2}$, tendo sido o número sempre aceito no intervalo de 5 a 15 . Com base no número de classes e no valor da amplitude, cal cul ou-se o val or de cada interval o de classes (IC) como sendo IC $=A / I$. Depois, foi calculada a freqüência de lavouras de alta produtividade, pertencentes a cada classe definida pela amplitude e interval o de classes. Com os val ores de freqüência de lavouras em cada classe (f), cal culou-sea Chance Matemática (E') para a produção em cada uma dessas classes, pela seguinte expressão: $E^{\prime}=f . P ;$ em que $P=$ produtividade média das lavouras de alta produtividade, cujos teores foliares pertenciam à respectiva classe (Wadt et al., 1994). As classes de concentração do nutriente de maiores val ores para a Chance Matemática foram consideradas como a faixa ótima e, para esta, determinou-se a respectiva mediana, considerada como o nível ótimo do nutriente, eo limite inferior da faixa ótima foi considerado como sendo o nível crítico do nutriente.

\section{RESULTADOS E DISCUSSÃO}

\section{Análise estatística dos índices DRIS}

Uma das vantagens da utilização dos índices DRIS está na possibilidade de explicar parte da variabilidade da produção de uma planta de forma mais adequada que pela utilização dos teores dos nutrientes tomados isoladamente (Sumner, 1977), desde que, em condições de campo, em decorrência dos efeitos de diluição e de concentração dos nutrientes (J arrel \& Beverly, 1981), orelacionamento entre a concentração do nutriente e a produção vegetal torna-se fracamente definido.

Embora seja possível estimar uma curva de resposta ideal entre a produtividade do talhão florestal e o teor de dado nutriente numa parte da árvore (Figura 1 ), sua utilidade prática pode ser duvidosa. Fazendo o gráfico do incremento médio anual (I MA) ea concentração deK nas fol has, poderse-ia traçar uma curva de resposta ideal $\left(C_{i}\right)$, que, apesar de estabelecer uma tendência entre a produtividadee a concentração deK, não possibilita definir em que grau o teor de K estaria realmente limitando a produtividade, já que a variação do teor de $\mathrm{K}$ foi praticamente o mesmo em qualquer nível de produtividade das árvores (Figura 1).

Os índices DRIS normalmente conduzem a um melhor relacionamento entre a produtividade do talhão e o estado nutricional da árvore em rel ação ao nutriente analisado (Beaufils, 1973), relacionamento este que pode ser representado pela dispersão dos pontos em forma de um triângulo (árvore de natal), quando a produtividade (variável dependente) é colocada em função do índice DRIS do nutriente (variável independente) (Figura 2). Esse modelo de dispersão indica que, quanto maior o módulo do índices DRIS, menor a média da produtividade dos talhões florestais (Figura 2).

O IBN apresentou um comportamento bastante próximo do modelo teórico, como, por exemplo, a dispersão do I BN dos macronutrientes da casca em relação à produtividade das árvores (Figura 3).

Contudo, a validação dos resultados dos índices DRIS não pode basear-se somente na anál ise gráfica (Figuras 2 e 3). Uma alternativa para a validação desses resultados consiste em verificar se a freqüência com que os nutrientes ocorrem como os mais limitantes foi atribuída ou não ao acaso. Caso tenha sido atribuída ao acaso, significa que os índices DRIS não devem ser utilizados na avaliação nutricional das árvores de eucalipto.

Considerando que os fatores responsáveis pelas diferenças de produtividade entregrupos de árvores com baixo ou al to crescimento sejam os nutricionais, torna-se possível imaginar que a freqüência com que os nutrientes ocorrem como os mais limitantes nas árvores pertencentes à dasse de média produtividade não seria a mesma daquela observada para as árvores de baixa e de alta produtividade.

A análise destas duas hipóteses, pelo teste de quiquadrado, revel ou que a freqüência com que cada nutriente ocorreu como o mais limitante não foi ao acaso, diferindo entre as classes de média e de baixa ou de alta produtividade (Quadro 1). 


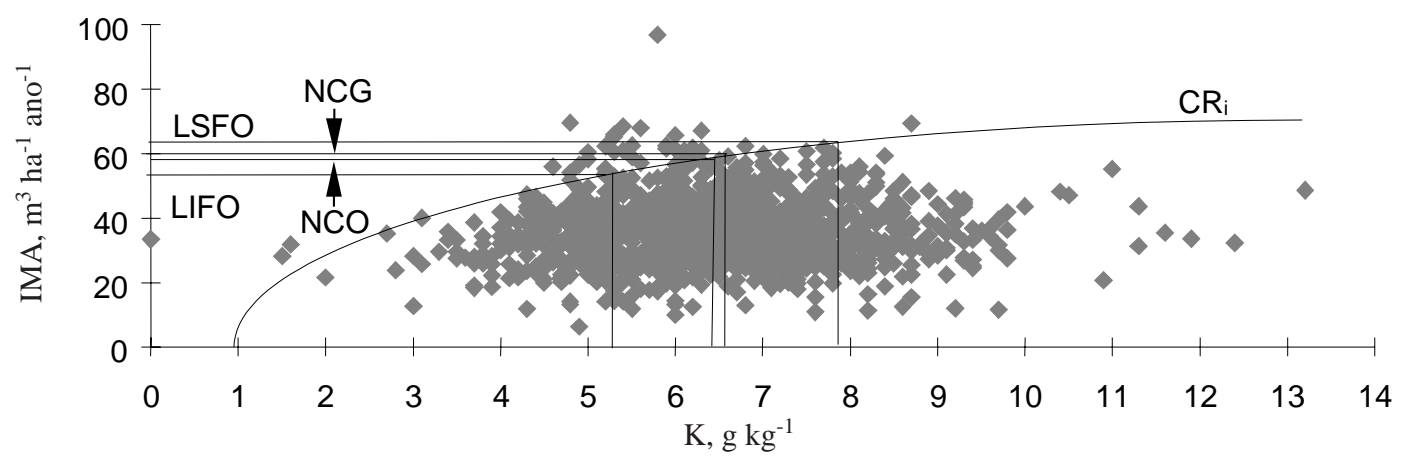

Figura 1. Gráfico de dispersão entre o incremento médio anual do lenho (IMA) em relação ao teor de $K$ nas folhas de 1.213 árvores de eucalipto. $C_{R_{i}}=$ curva de resposta ideal, $\mathbf{N C G}=$ nível crítico gráfico; NCO = nível crítico ótimo; LIFO = limite inferior da faixa ótima e; LSFO = limite superior da faixa ótima.

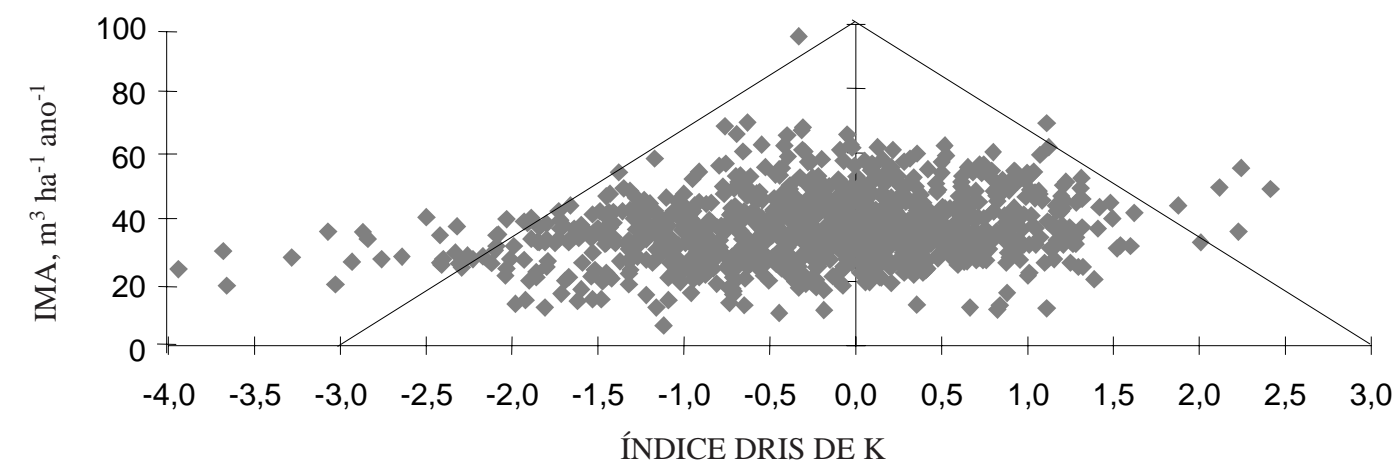

Figura 2. Gráfico de dispersão entre o incremento médio anual do lenho (IMA) em relação ao índice DRIS de K nas folhas de $\mathbf{1 . 2 1 3}$ de árvores adultas de eucalipto.

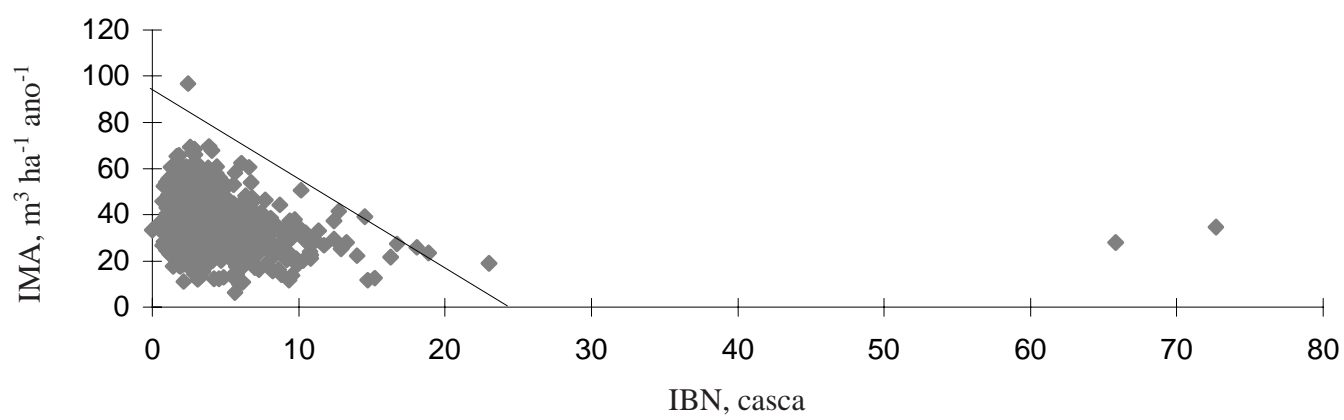

Figura 3. Gráfico de dispersão para o incremento médio anual do lenho (IMA) em relação ao índice de balanço nutricional (IBN) da casca de $\mathbf{1 . 2 1 3}$ árvores adultas de eucalipto.

\section{Determinação dos valores dereferência pelo DRIS}

Os dois critérios utilizados para a determinação dos valores de referência dos teores dos nutrientes apresentam estreita concordância; os val ores obtidos pelo critério do NCG foram, na maioria das vezes, superiores aos obtidos pelo critério do NCO (Quadro 2). As duas exceções foram oNCG deK nos gal hos e de $\mathrm{S}$ nas fol has.
De modo geral, os valores para NCG e NCO aproximaram-se daqueles que seriam determinados pela $\mathrm{CR}_{\mathrm{i}}$, se o valor crítico fosse considerado como sendo a concentração do nutriente equival entea $90 \%$ da produtividade máxima determinada pela curva de resposta ideal (Figura 1).

Os coeficientes de determinação $\left(R^{2}\right)$ entre teor do nutriente e seu respectivo índice DRIS, em sua 
Quadro 1. Freqüências observada e esperada (FE), em número de casos, para a ocorrência de $\mathbf{N}, \mathbf{P}$, $\mathrm{K}, \mathrm{Ca}$, Mge $S$ como os nutrientes mais limitantes por deficiência e níveis de significância para as hipóteses nula "N1"e "N2", segundo as classes de produtividade de árvores de eucalipto

\begin{tabular}{|c|c|c|c|c|c|c|c|c|c|}
\hline \multirow{2}{*}{ Classe } & \multicolumn{6}{|c|}{ Freqüência observada } & \multirow{2}{*}{ FE } & \multirow{2}{*}{ N1 } & \multirow{2}{*}{$\mathbf{N} 2$} \\
\hline & $\mathbf{N}$ & $\mathbf{P}$ & $\mathbf{K}$ & $\mathrm{Ca}$ & Mg & $\mathbf{S}$ & & & \\
\hline & \multicolumn{7}{|c|}{ Folha } & \multicolumn{2}{|c|}{ - \% - } \\
\hline $\begin{array}{l}A \\
B \\
M\end{array}$ & $\begin{array}{l}26 \\
34 \\
36\end{array}$ & $\begin{array}{l}64 \\
47 \\
65\end{array}$ & $\begin{array}{r}74 \\
129 \\
137\end{array}$ & $\begin{array}{l}66 \\
43 \\
86\end{array}$ & $\begin{array}{r}53 \\
87 \\
102\end{array}$ & $\begin{array}{l}58 \\
49 \\
65\end{array}$ & $\begin{array}{l}57 \\
65 \\
82\end{array}$ & $\begin{array}{l}0,5 \\
0,5 \\
0,5\end{array}$ & $\begin{array}{l}0,5 \\
0,5 \\
\text { n.d. }\end{array}$ \\
\hline $\begin{array}{l}A \\
B \\
M\end{array}$ & $\begin{array}{l}42 \\
82 \\
91\end{array}$ & $\begin{array}{l}86 \\
46 \\
71\end{array}$ & $\begin{array}{r}48 \\
116 \\
103\end{array}$ & $\begin{array}{c}\text { Galho } \\
48 \\
46 \\
71\end{array}$ & $\begin{array}{l}61 \\
47 \\
86\end{array}$ & $\begin{array}{l}52 \\
69\end{array}$ & $\begin{array}{l}57 \\
65 \\
82\end{array}$ & $\begin{array}{l}0,5 \\
0,5 \\
5,0\end{array}$ & $\begin{array}{l}0,5 \\
0,5 \\
\text { n.d. }\end{array}$ \\
\hline $\begin{array}{l}A \\
B \\
M\end{array}$ & $\begin{array}{l}39 \\
60 \\
61\end{array}$ & $\begin{array}{r}98 \\
69 \\
106\end{array}$ & $\begin{array}{l}60 \\
94 \\
88\end{array}$ & $\begin{array}{c}\text { Casca } \\
47 \\
72 \\
102\end{array}$ & $\begin{array}{l}40 \\
38 \\
62\end{array}$ & $\begin{array}{l}56 \\
55 \\
71\end{array}$ & $\begin{array}{l}57 \\
65 \\
82\end{array}$ & $\begin{array}{l}0,5 \\
0,5 \\
0,5\end{array}$ & $\begin{array}{l}0,5 \\
0,5 \\
\text { n.d. }\end{array}$ \\
\hline $\begin{array}{l}A \\
B \\
M\end{array}$ & $\begin{array}{l}48 \\
66 \\
79\end{array}$ & $\begin{array}{l}25 \\
50 \\
50\end{array}$ & $\begin{array}{l}54 \\
57 \\
66\end{array}$ & $\begin{array}{l}\text { enh } \\
42 \\
40 \\
53\end{array}$ & $\begin{array}{r}109 \\
93 \\
148\end{array}$ & $\begin{array}{l}82 \\
94\end{array}$ & $\begin{array}{l}57 \\
65 \\
82\end{array}$ & $\begin{array}{l}0,5 \\
0,5 \\
0,5\end{array}$ & $\begin{array}{l}0,5 \\
0,5 \\
\text { n.d. }\end{array}$ \\
\hline
\end{tabular}

n.d. = não determinado. N1 = hipótese nula 1 (freqüência dos nutrientes como mais limitantes atribuída ao acaso). N2 = hipótese nula 2 (freqüência dos nutrientes como mais limitantes foi igual entre as subpopulações de média produtividade com as demais subpopulações). mai oria, foram baixos (Quadro 2). Considerando que o valor do NCG foi obtido, igualando-se a variável dependentea zero na regressão pol inomial ajustada, conforme se ilustra para K nas fol has (Figura 4), a exatidão da determinação do NCG poderia ser comprometida pela imprecisão da equação ajustada. Somente os coeficientes de determinação para P na casca e para Mg no lenho foram maiores que 0,90 e, além desses dois, somente para $P$ nos gal hos e para $\mathrm{K}$ e Ca nas folhas observaram-se coeficientes de determinação maiores que 0,70 . I sso significa que apenas $20 \%$ das regressões mostraram coeficientes de determinação superiores a 0,70 (Quadro 2).

O fato de o NCO ter resultado, sistematicamente, em menores val ores que o NCG indica que o primeiro deveria ser preferencialmente adotado, já que seria menor a probabilidade de diagnosticar como deficientes árvores que estariam sofrendo efeitos de diluição por apresentarem taxa de crescimento superior à taxa de acumulação do nutriente.

Contudo, deve-se destacar que os valores encontrados para NCG e NCO foram bastante próximos daqueles citados pela literatura e obtidos da forma convencional (Quadro 2). Também para os nutrientes no solo, Oliveira \& Sousa $(1988,1993)$ observaram que o DRIS foi capaz de determinar corretamente os níveis de suficiência.

Quadro 2. Faixa ótima, mediana da faixa ótima, valor crítico ótimo (NCO), valor crítico gráfico (NCG), coeficiente de determinação entre o índice DRIS e os teores dos nutrientes $\left(R^{2}\right)$ e valores-padrões publicados para a concentração dos macronutrientes em órgãos de árvores adultas de eucalipto

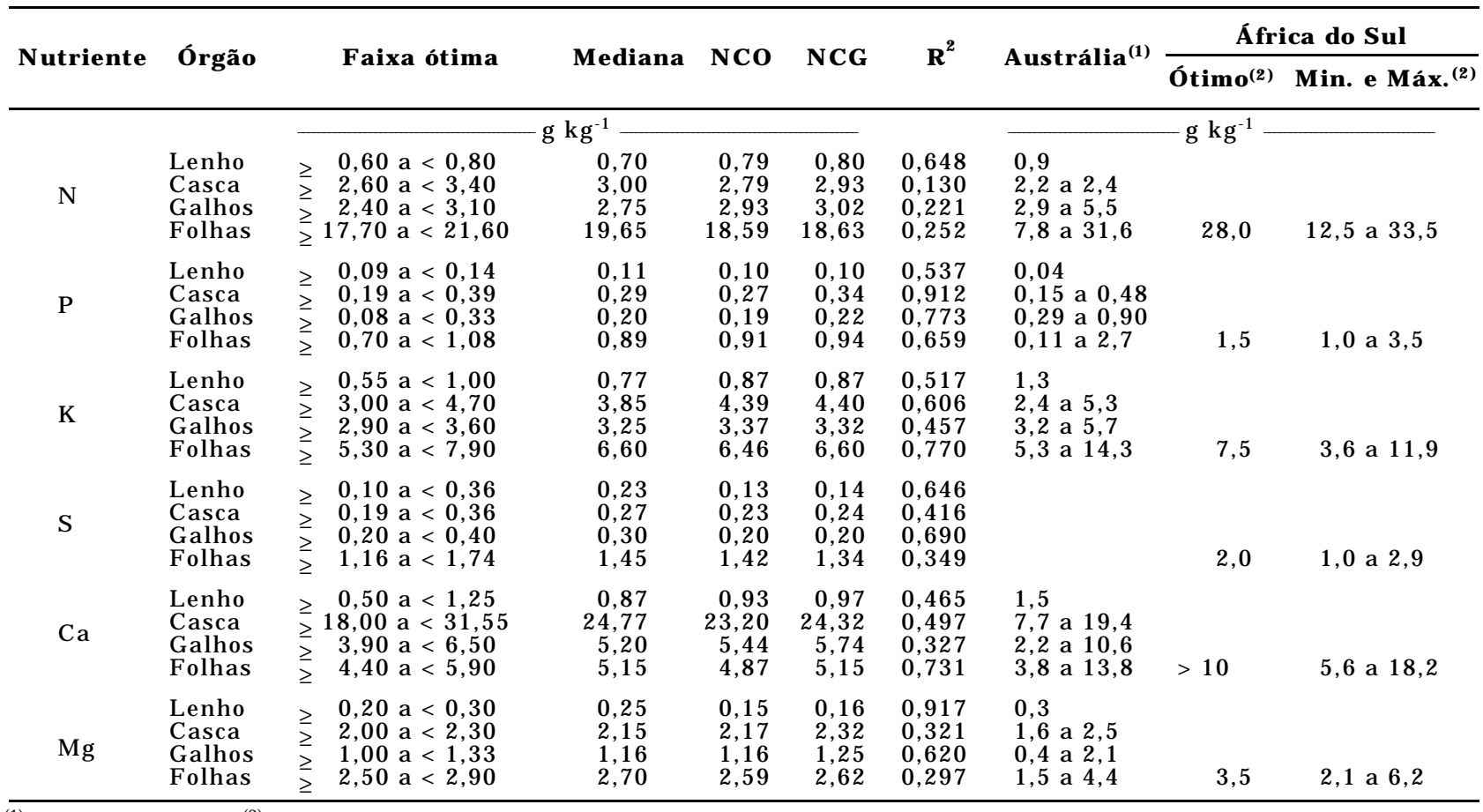

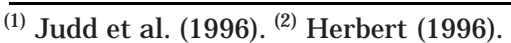




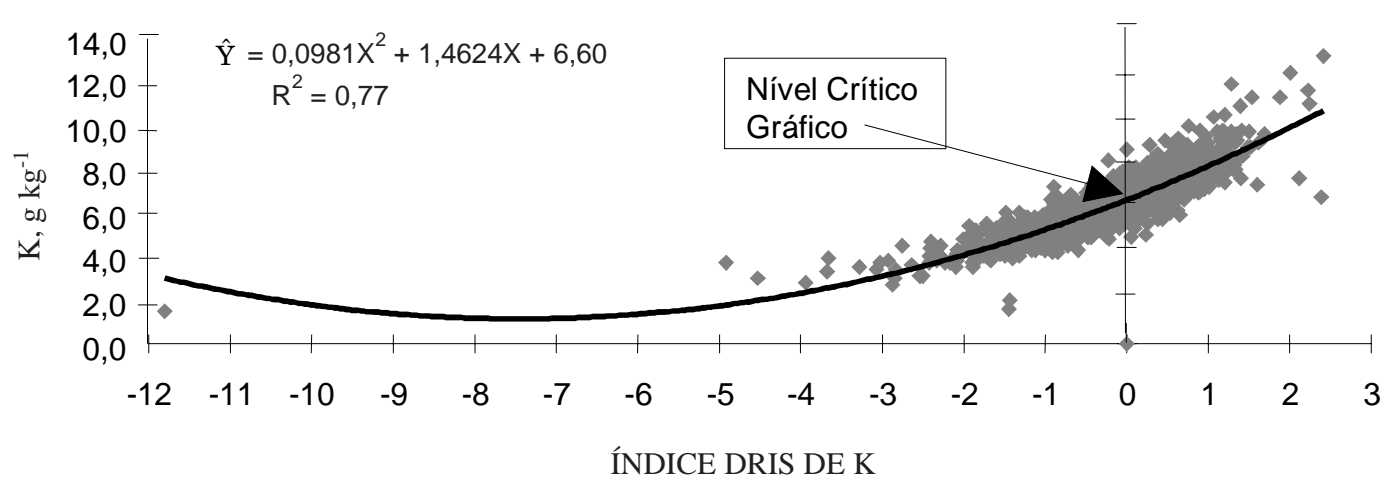

Figura 4. Gráfico de dispersão e regressão quadrática entre o teor de K em relação ao índice DRIS de K nas folhas de 1.213 árvores adultas de eucalipto.

\section{Determinação dos valores de referência pela Chance Matemática.}

De maneira geral, a amplitude da faixa ótima determinada pela Chance Matemática foi menor que a variação observada para os teores dos nutrientes em plantações de Eucalyptus grandis na Austrália (J udd et al., 1996) e na África do Sul (Herbert, 1996) (Quadro 2). As exceções foram a amplitude da faixa ótima de $\mathrm{N}$ na casca, de $\mathrm{P}$ nos galhos e de Ca na casca que esteve acima ( $\mathrm{N}$ e Ca na casca) ou abaixo ( $P$ nos galhos) dos limites observados para a concentração desses nutrientes em eucaliptos na Austrália (J udd et al., 1996). Também as amplitudes das faixas ótimas para $\mathrm{P}$ e para $\mathrm{Ca}$ nas folhas estiveram abaixo dos valores mínimos observados para esses nutrientes na África do Sul (Herbert, 1996).

Constata-se que o limite superior da faixa ótima deN,P,S, Ca eMg mostrou-seinferior aos valoresótimos para a concentração dos macronutrientes em fol has de eucalipto publicados por Herbert (1996), o que evidencia a mai or eficiência nutricional dos híbridos de Eucalyptus grandis x Eucalyptus urophylla em relação às árvores de Eucalyptus grandis. Além disso, os híbridos de $\mathrm{E}$. grandis X E. urophylla apresentaram maiores val ores para a concentração dos nutrientes na casca e menores nas folhas, em comparação com as árvores de E. grandis, o que foi particularmente válido para N e para Ca (Quadro 2).

\section{Estimativa do nível crítico e do coeficiente de utilização biológica}

Ambos os métodos (DRIS e Chance Matemática) mostraram-se promissores para a determinação dos valores de referência para o teor dos nutrientes no eucalipto.

A determinação do val or ótimo para o coeficiente de utilização biológica (CUB) poderia, portanto, vir a ser feita com qualquer um dos métodos, obtendose resultados praticamente idênticos. O NCO deve ser preferido em relação ao NCG, pois com o primeiro há menor probabilidade de superestimar as quantidades de nutrientes demandadas pelas árvores e a mediana da faixa ótima poderia ser utilizada para obtenção do nível ótimo e, por conseqüência, do coeficiente de utilização biológica (CUB) (Barros \& Novais, 1996), caso em que o CUB seria numericamente equivalente ao inverso da mediana da faixa ótima.

O limite inferior da faixa ótima parece ser mais indicado para estimar o nível crítico dos nutrientes, ou seja, daquela concentração abaixo da qual se faz necessária a aplicação de fertilizantes. A principal vantagem estaria, neste caso, na possibilidade de determinar o nível crítico para os nutrientes em árvores de eucalipto a partir de informações de monitoramento nutricional de talhões comerciais, sem necessi dade do estabel ecimento de experimentos de calibração.

Deforma semelhanteaosugerido para Pinus taeda L. (Needham et al., 1990) e para Coffea canephora (Novais et al., 1994), os métodos DRIS e Chance Matemática mostraram-se promissores para determinar os valores-padrões de referência para a concentração dos nutrientes em árvores adultas de eucal ipto. A vantagem da utilização desses métodos está na possibilidade de aproveitar dados provenientes de monitoramento nutricional de árvores detal hões comerciais, sem a necessidade de estabel ecer experimentos de cal ibração. E seriam de pouca utilidade a longo prazo, desde que a própria evol ução do material genético exi giria a reinstal ação destes experimentos periodicamente. Além disso, com a utilização dos métodos DRIS e Chance Matemática, o tempo para a estimativa dos valores de referência limita-se ao período envol vido entre o abatimento das árvores, amostragem dos tecidos para análise química, execução da própria análise química e a interpretação dos resultados, o que pode ser feito num prazo de poucos meses. Comparativamente, somente a condução do ensaio de cali ibração, no período que antecede o abate das árvores, poderia levar de 36 a 72 meses, em média. 


\section{CONCLUSÕES}

1. Os métodos da Chance Matemática e do DRIS mostraram-seadequados para estimar os valores de referência para os macronutrientes em árvores de eucalipto.

2. O método da Chance Matemática foi indicado para estimar o nível crítico, o nível ótimo e o coeficiente de utilização biológica.

3. O método do DRIS foi indicado para determinar o nível crítico, sendo o critério do nível crítico ótimo mais adequado que o do nível crítico gráfico.

\section{LITERATURA CITADA}

BARROS, N.F. \& NOVAIS, R.F. Eucalypt nutrition and fertilizer regimes in Brazil. In: ATTIWILL, P.M. \& ADAMS, M.A. eds. Nutrition of Eucalypts. Collingwood, CSIRO, 1996. p.335-355.

BEAUFILS, E.R. Diagnosis and Recommendation Integrated System (DRIS). Pietermaritzburg, University of Natal, 1973. 132p. (Soil Sci. Bull, 1)

BHARGAVA, B.S. \& CHADHA, K.L. Leaf nutrient guide for fruit and plantation crops. Fert. News, v.33, n.7, p.21-29, 1988.

JARREL, W.M. \& BEVERLY, R.B. The dilution effect in plant nutrition studies. Adv. Agron., 34:197-224, 1981.

J ONES, C.A. Proposed modifications of the Diagnosis and Recommendation Integrated System (DRIS) for interpreting plant analyses Comm. Soil Sci. Plant Anal., 12:785-794, 1981.

J UDD, T.S.; ATTIWILL, P.M. \& ADAMS, M.A. Nutrient concentration in Eucalyptus: A synthesis in relation to differences between taxa, sites and components. In: ATTIWILL, P.M. \& ADAMS, M.A., eds. Nutrition of Eucalypts. Collingwood, CSIRO, 1996. p.123-153.
HERBERT, M.A. Fertilizers and eucalypt plantations in South Africa. In: ATTIWI LL, P.M. \& ADAMS, M.A., eds. Nutrition of Eucalypts. Collingwood, CSIRO, 1996. p.303-325.

NEEDHAM, T.D.; BURGER, J.A. \& ODERWALD, R.G. Relationship between Diagnosis and Recommendation Integrated System (DRIS) optima and foliar nutrient critical levels. Soil Sci. Soc. Am. J., 54:883-886, 1990.

NOVAIS, R.F.; WADT, P.G.S.; ALVAREZ V., V.H. \& BARROS, N.F. Levantamento do estado nutricional de cafeei ros do Estado do Espírito Santo com base no método da Chance Matemática. In: REUNIÃO BRASILEIRA DE FERTILIDADE DO SOLO E NUTRIÇÃO DE PLANTAS, 21., Petrolina, 1994. Anais. Petrolina, SBCS/EMBRAPACPATSA, 1994. p.182-183.

OLIVEIRA, S.A. \& SOUSA, D.M.G. Interpretação da análise de solo pelo método DRIS (Sistema Integrado de Diagnose e Recomendação). In: REUNIÃO BRASILEIRA DE FERTILIDADE DO SOLO E NUTRIÇÃO DE PLANTAS, 18., Guarapari, 1988. Resumos. Vitória, SEAG-ES/ EMCAPA/EMATER-ES/SBCS, 1988. p.47-48.

OLIVEIRA, S.A. \& SOUSA, D.M.G. Uso do DRIS modificado na interpretação de análise de solo para a soja no leste de Mato Grosso. In: CONGRESSO BRASILEIRO DE CIÊNCIA DO SOLO, 24., Goiânia, 1993. Resumos, Goiânia, SBCS, 1993. v.2, p.83-84.

SUMNER, M.E. Use of the DRIS system in foliar diagnosis of crops at high yield levels. Comm. Soil Sci. Plant Anal., 8:251268, 1977.

WADT, P.G.S. Os métodos da Chance Matemática e do Sistema Integrado de Diagnosee Recomendação (DRIS) na avaliação nutricional de plantios de eucalipto. Viçosa, Universidade Federal de Viçosa, 1996. 99p. (Tese de doutorado).

WADT, P.G.S.; ALVAREZ V., V.H.; NOVAIS, R.F. \& BARROS, N.F. Método da Chance Matemática para a determinação das faixas infra-ótima, ótima e supra-ótima dos teores foliares de nutrientes. In: REUNIÃO BRASILEIRA DE FERTILIDADE DO SOLO E NUTRIÇÃO DE PLANTAS, 21., Petrolina, 1994. Anais. Petrolina, SBCS/EMBRAPACPATSA, 1994. p.186-187. 